\title{
Enhancing the lateral resolution in infrared microspectrometry by using synchrotron radiation: applications and perspectives
}

\author{
P. Dumas ${ }^{1}$, G.L. Carr ${ }^{2}$ and G.P. Williams ${ }^{2}$ \\ ${ }^{1}$ LURE, Centre Universitaire Paris-Sud, BP 34, 91898 Orsay Cedex, France \\ ${ }^{2}$ National Synchrotron Light Source, Brookhaven National Laboratory, Upton, NY 11973, USA
}

The use of a much brighter source than the conventional blackbody allows an enhancement in lateral resolution in microanalysis using infrared spectroscopy, which thus, becomes limited only by the diffraction criteria. Numerous new applications have been performed in various research fields, which highlight the usefulness of such a new analytical technique.

\section{Introduction}

The mid-infrared region (25-2.5 microns) is a very useful frequency range as most of the existing molecular groups have vibrational energies in this region. These intramolecular vibrational modes play an important role in analytical work, and are responsible for the widely recognized success of the infrared spectroscopic technique.

Moreover, infrared microspectroscopy [1,2] is a microanalytical and imaging technique which achieves contrast via the intramolecular vibrational modes which are often referred to as the "fingerprint" modes. In such an approach, it is always highly desirable to achieve as high a spatial resolution as possible, although diffraction provides the ultimate limit. Using a conventional infrared thermal source, the resolution cannot be made as low as the diffraction limit would allow. This is due to the lack of energy at the sample position when closing the aperture down (typically below 15 to 10 microns).

The use of synchrotron radiation has overcome those limitations [2,3]. Infrared synchrotron radiation is about 1000 times brighter than globar sources, and therefore offers considerably improved signal to noise capabilities. Synchrotron radiation is also a polarized and pulsed (on the 100 picosecond timescale) source, that opens up new perspectives in analytical chemistry.

In this article, we briefly describe the advantages of the synchrotron source. We will then present recent applications that illustrate the superiority of this technique, in various domains. As illustrated in figure 1, improving the lateral resolution is beneficial in three categories of experiments: (a) one can identify individual particles (Fig. 1a) to understand the degree of heterogeneity in distribution of compounds, as this will be exemplified in this article; (b) one can investigate the diffusion between two interfaces which is a major problem in many applications involving fabrication processes (Fig. 1b); and (c) one can record infrared spectra from a small area, and scan over a large domain (Fig. 1c), to obtain images of small samples using chemical contrast techniques. We will show how this last experimental scheme has been successfully used in Biology and Petrography.
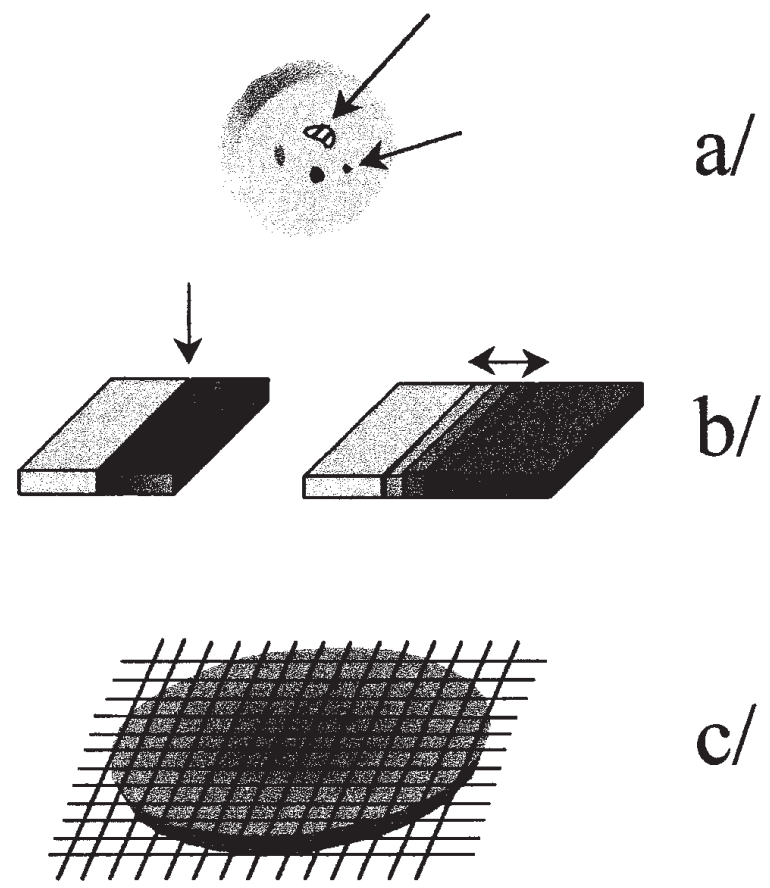

$C /$

Figure 1. Analysis involving improved lateral resolution, as that offered by the use of a synchrotron source, can be classified in three categories: a) Identification of individual particles, of micron-size b) Detailed analysis of a concentration profile between two interfaces, or along a material and c) chemical imaging with high contrast. 


\section{Synchotron radiation and infrared microspectrometry}

As a light source for spectroscopy, synchrotron radiation (SR) has 2 key properties, namely high brilliance, and a continuous spectral distribution of the intensity, over the entire energy range. Synchrotron infrared radiation can be extracted either from a bending magnet [3], or from an undulator [4]. In general, large collecting angles are required. Some 15 beamlines now exist around the world, most of them dedicated to microscopy.

The first beamline to run large programs in infrared spectroscopy was built at the National Synchrotron Light Source, Brookhaven National Laboratory [2]. More recently, a new beamline has been opened at SUPERACO France (beamline MIRAGE - port SA5), which is dedicated to infrared microspectroscopy.

Figure 2 shows a schematic of an infrared microscope. In this set up, the synchrotron beam replaces the conventional

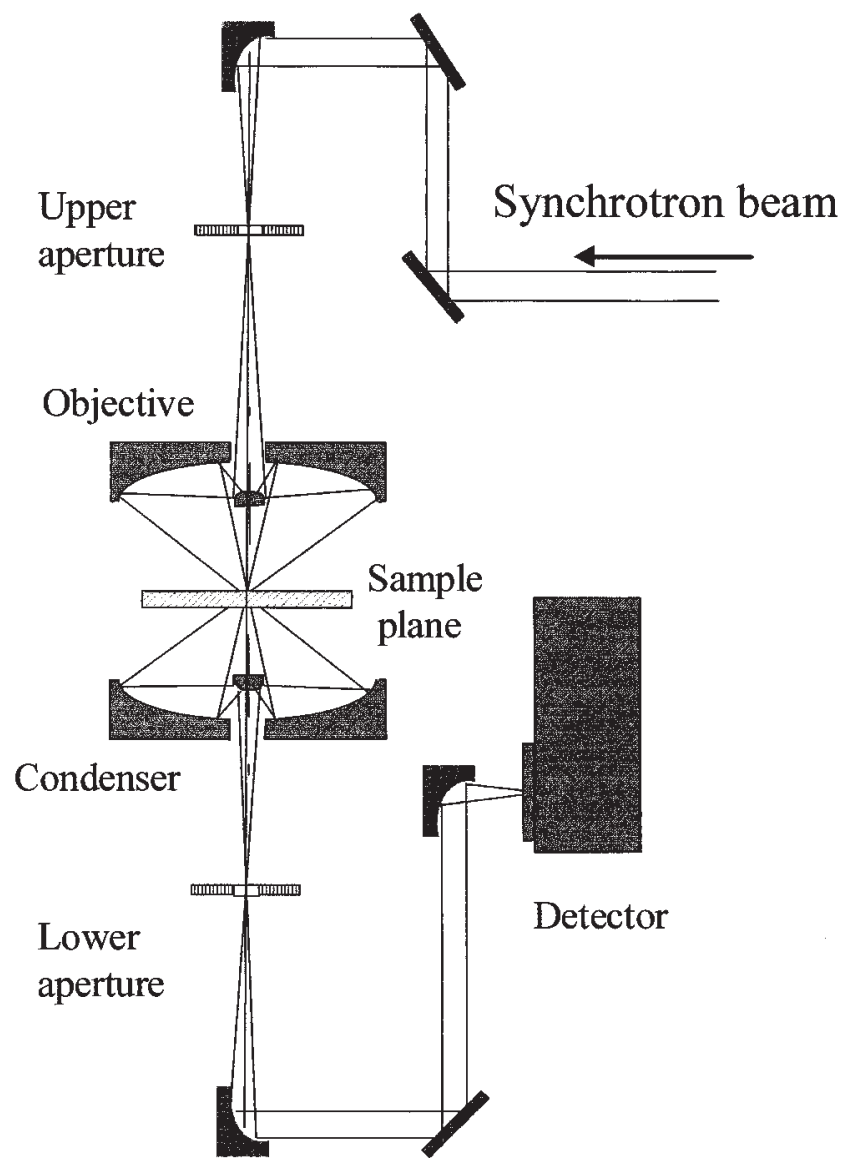

Figure 2. Schematic of an IR doubly confocal microspectrometer, coupled to synchrotron radiation. The beam is focused onto the sample via Schwartzschild mirrors, and the sample can be scanned to produce a map. blackbody source. The size of the upper aperture, which is further projected onto the sample plane by the Schwarzschild objective, determines the lateral resolution. In conventional IR microscopy, the size of this aperture is limited, due to the weakness of the infrared source. Figure 3 illustrates the advantage of using a synchrotron source. When using a conventional source, with a $3 \times 3 \mu \mathrm{m}^{2}$ aperture, almost no signal is detected, thus producing an enormous noise (Fig. 3a). With such experimental conditions, analysis of small domain size is precluded. The synchrotron source is a more appropriate source, as a large signal is detected even for this small aperture (the gain in brightness, for the MIRAGE beamline in Orsay, and for a $3 \times 3 \mu \mathrm{m}^{2}$ aperture, is about 800). Figure $3 \mathrm{~b}$ displays the $100 \%$ line obtained for 100 scans at $4 \mathrm{~cm}^{-1}$ resolution (recording time of about 60 seconds). The inset of this figure shows an enlargement of the $2500-3000 \mathrm{~cm}^{-1}$ frequency region. A very good signal to noise is achieved. This demonstrates the superiority of using a synchrotron source in infrared microspectrometry. In order to demonstrate the analytical advantage of synchrotron infrared microspectrometry, the
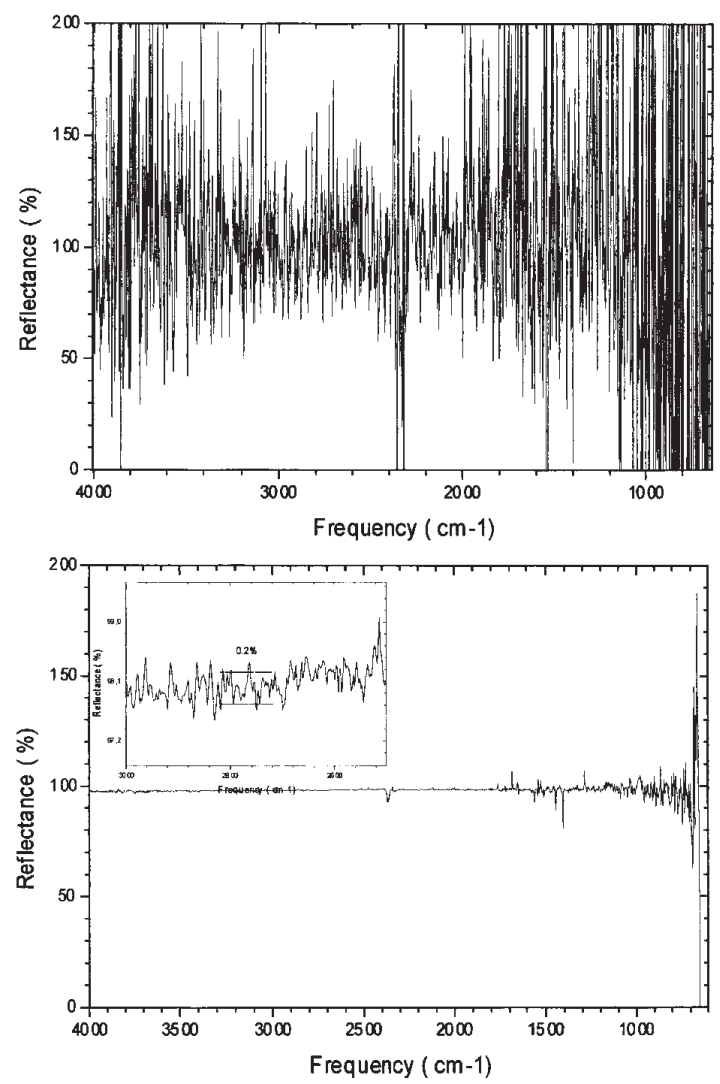

b/

Figure 3. Signal to Noise spectra obtained after coadding 100 scans at $4 \mathrm{~cm}^{-1}$ resolution. a) for the globar (internal) source and b) with the synchrotron radiation. Shown as insert in spectrum $b$ / is an enlargement of the $2500-3000 \mathrm{~cm}^{-1}$ frequency region: the $\mathrm{S} / \mathrm{N}$ is $0.2 \%$. The spectra have been acquired at the MIRAGE beamline, SUPERACO. 
spectrum of a small polymer particle (about $4 \mu \mathrm{m}$ size) was recorded, with an aperture of $3 \times 3 \mu \mathrm{m}^{2}$, both with the globar and the synchrotron sources. Figure 4 displays the spectra recorded in both cases, demonstrating the analytical superiority of the synchrotron source.

To understand better the brightness advantage, figure 5 shows the intensity profile of the intrinsic spotsize of the synchrotron source projected onto the sample stage using a $32 \times$ objective. Due to the high localization of the intensity, closing the aperture down does not affect drastically the number of probing infrared photons. However, with such small working apertures, the diffraction issue becomes important. G.L. Carr et al. have shown that diffraction starts when apertures reach $3 / 4$ of the wavelength [5]. These effects have to be taken into account, especially when relative concentration of compounds is of interest, and the two characteristic frequencies are separated by few hundreds of wavenumbers.

\section{Applications of synchotron infrared microspectrometry}

Many applications have already been tried, including measurements of individual extraterrestrial particles, semiconductor materials and devices, biological samples - (which have also involved cosmetics, and prosthetics), polymer, and geological samples. For illustrative purposes, we show examples in Astrophysics, Biology and Geology.

\section{Extraterrestrial particles}

The potential of the analysis of extraterrestrial particles for understanding the formation and evolution of the Solar System is highly dependent on the capability of correlating all compositional measurements (elemental, isotopic, molecular and mineralogical) at the grain size scale. In that framework, synchrotron IR microscopy appears as a technique of choice for providing the mineralogical and molecular content of grains [6].

Figure 6 shows the IR spectrum of two micron-size interplanetary dust particles (received from NASA). These particles are known to be rich in $\mathrm{Fe}$ and $\mathrm{S}$, with minor amounts of $\mathrm{Mg}, \mathrm{Si}$ and $\mathrm{Ni}$. One can notice the silicate signatures at 1010 and $890 \mathrm{~cm}^{-1}$, the $\mathrm{CH}_{2}, \mathrm{CH}_{3}$ stretching modes in the $2800-3000 \mathrm{~cm}^{-1}$ region, and the presence of molecular water (broad band centered at $3300 \mathrm{~cm}^{-1}$ ). For comparison, the spectrum of the same particle taken with a blackbody source, with a $12 \times 12$ micron aperture, is displayed, highlighting the striking advantage of the synchrotron source.

\section{Geology}

Inclusions are commonly found in certain kinds of rocks. They consist of a void in the rock, filled with liquid, gas,

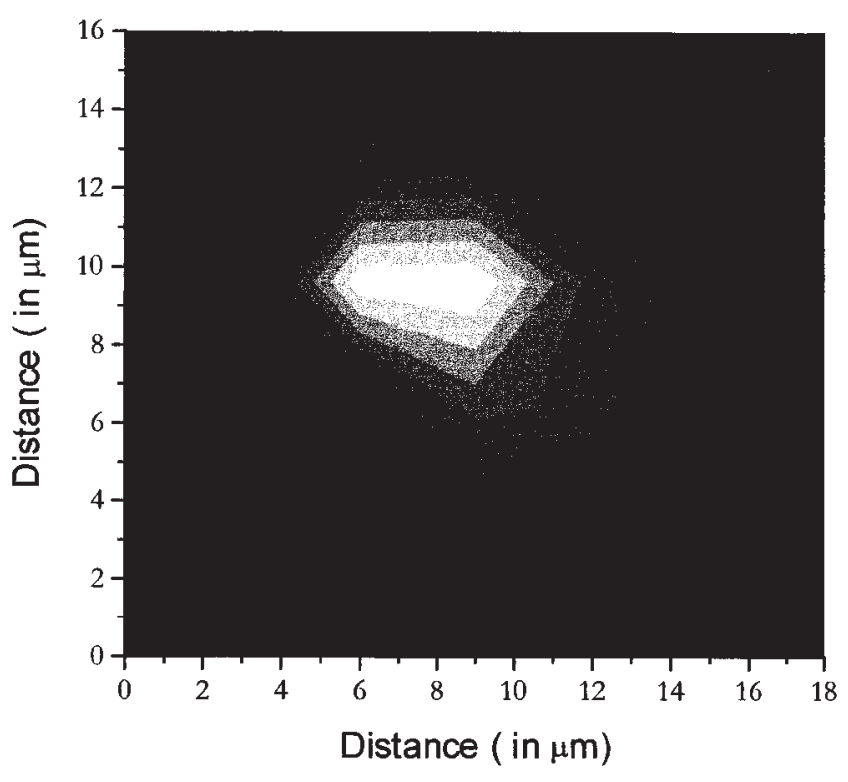

Figure 4. Intensity profile map of the intrinsic spotsize at the sample focus, using the synchrotron IR source. This profile has been recorded at the MIRAGE beamline (SUPERACO).

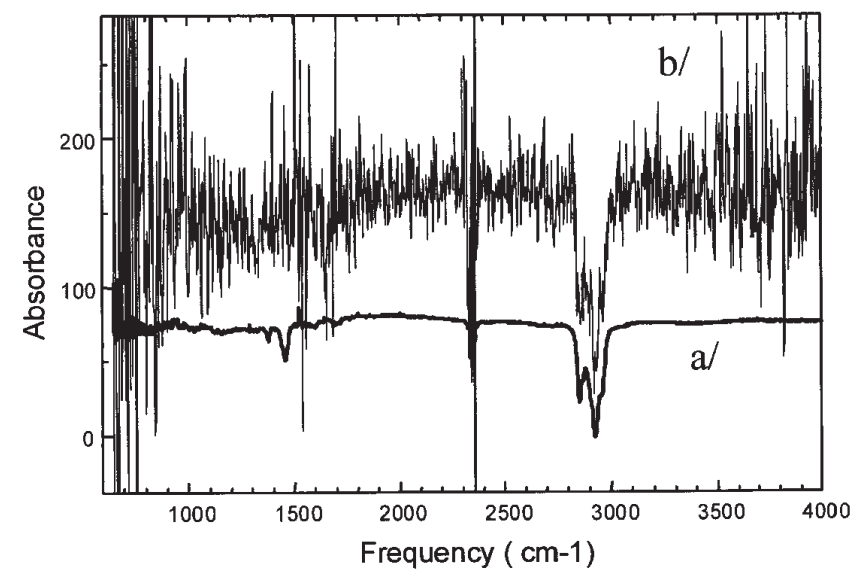

Figure 5. Infrared spectra of the same polymer particle (about 3 micron size), recorded with an aperture of $3 \times 3 \mu \mathrm{m}^{2}$, and using a) the synchrotron source and b) the globar source.

or both. Figure 7 shows a photo of such an inclusion (top left), together with a spectrum (center), taken using a $6 \times 6 \mu \mathrm{m}^{2}$ aperture. This spectrum shows various $\mathrm{CH}$ bands due to the presence of oil, while there is also a strong peak due to $\mathrm{CO}_{2}$, and a small peak due to $\mathrm{OH}$. Maps of the distribution of the $\mathrm{OH}, \mathrm{CH}_{2}$ and $\mathrm{CO}_{2}$ modes are also shown in figure 7 , and clearly reveal that the bubble evident in the photograph is composed of $\mathrm{CO}_{2}$ immersed in oil with uniformly distributed hydroxyl groups - probably at the fluid/solid interface. The pressure of $\mathrm{CO}_{2}$ and the nature of the oil can be discerned from the spectra, yielding valuable information about the origin of the rock sample and its potential as an oil source. 


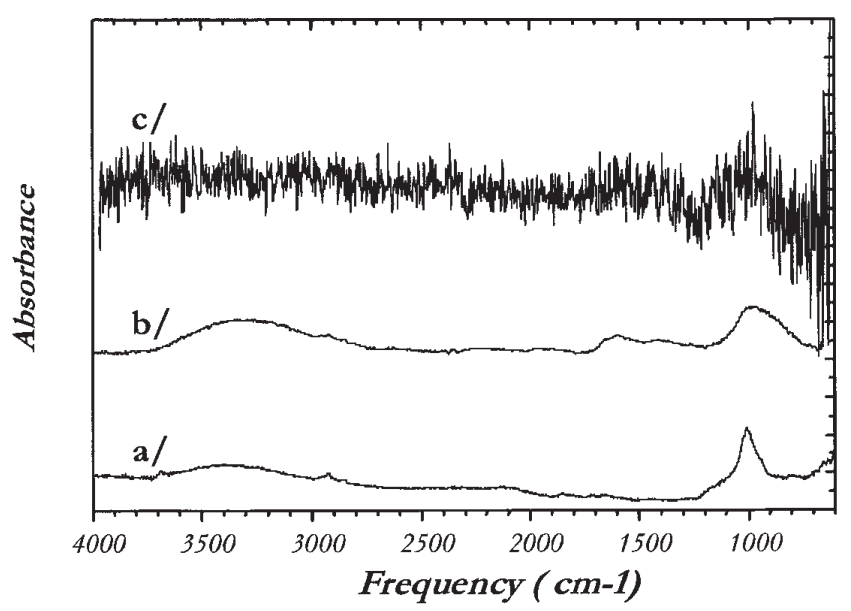

Figure 6. Infrared spectrum of two micron-size IDP particle (a) and b)). The spectra were taken with a $3 \times 3 \mu \mathrm{m}^{2}$ aperture. For comparison, spectrum c) is taken on the same particle than spectrum b), but with a globar source and an aperture of $12 \times 12$ $\mu \mathrm{m}^{2}$. They correspond to 128 accumulations at $4 \mathrm{~cm}^{-1}$ resolution. Synchrotron IR spectra have been taken at U4IR beamline (NSLS).

\section{Biology}

Biological materials contain molecules with a rich selection of intramolecular vibrational modes due to proteins, lipids and nucleic acids, yielding spectra that are similar to the one displayed in figure 8. More details can also be found in D.A. Wetzel's article, in this issue. This spectrum was taken on a living individual cell, with an aperture of $3 \times 3 \mu \mathrm{m}^{2}$. The dominant features in the spectra are the broad bands from 2 500-3 $500 \mathrm{~cm}^{-1}$, which arise from $\mathrm{NH}$ and $\mathrm{OH}$ groups, proteins and polysaccharides $\left(3063-3290 \mathrm{~cm}^{-1}\right)$ and both the symmetric and antisymmetric stretching modes of methylene $\left(\mathrm{CH}_{2}\right)$ and methyl $\left(\mathrm{CH}_{3}\right)$ groups (2 850$2960 \mathrm{~cm}^{-1}$ ) in lipids and proteins. The other main modes originate from CO-NH vibrations (amide I at $1650 \mathrm{~cm}^{-1}$ and amide II at $1540 \mathrm{~cm}^{-1}$ ). Sharper modes occur below these bands due to $\delta$-CH vibrations (1 450 and $\left.1390 \mathrm{~cm}^{-1}\right)$ and there is an amide III band at $1238 \mathrm{~cm}^{-1}$. In addition to these modes, and depending on the particular sample, there are lower frequency modes due to phosphodiester $\left(\mathrm{PO}_{2}\right)$ groups (1 $\left.240 \mathrm{~cm}^{-1}\right)$ in nucleic acids, and carbonyl groups (CO) in phospholipids at $1740 \mathrm{~cm}^{-1}$.

Synchrotron infrared microspectrometry has demonstrated for the first time that the vibrational modes described above could be used to establish chemical images of proteins, lipids and nucleic acids in living biological cells, without the

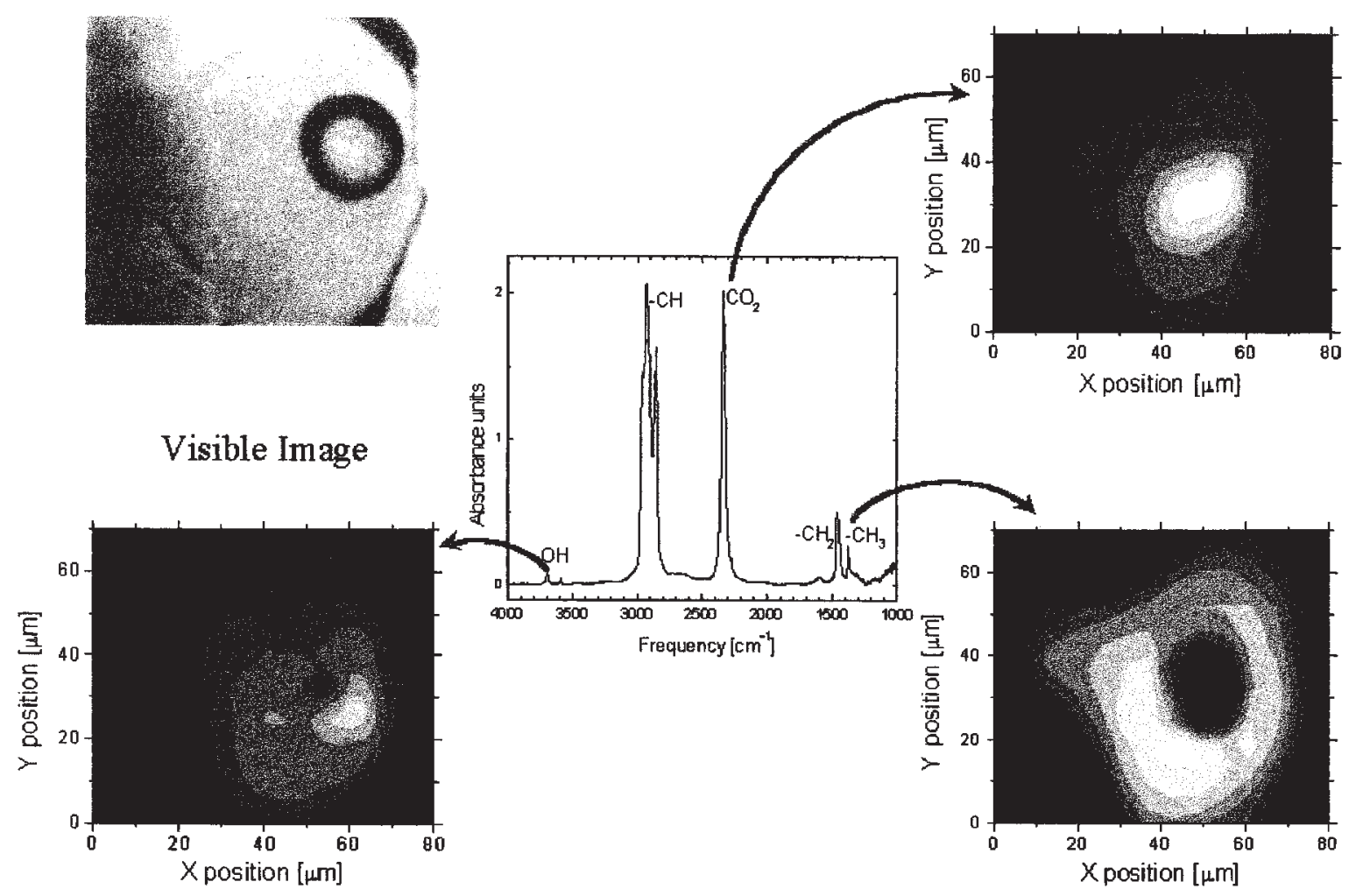

Figure 7. Infrared microspectroscopy of a fluid inclusion (Optical image, top left). Center displays one characteristic spectrum recorded inside the inclusion, with a $3 \times 3 \mu \mathrm{m}^{2}$ aperture. The maps, corresponding to the spatial distribution of $\mathrm{OH}, \mathrm{CO}_{2}$ and $\mathrm{CH}_{\mathrm{n}} \mathrm{groups}$ are shown. One can see clearly that $\mathrm{CO}_{2}$ is separate from the oil content of this inclusion, and corresponds to the bubble observed on the optical image (from [11]). 


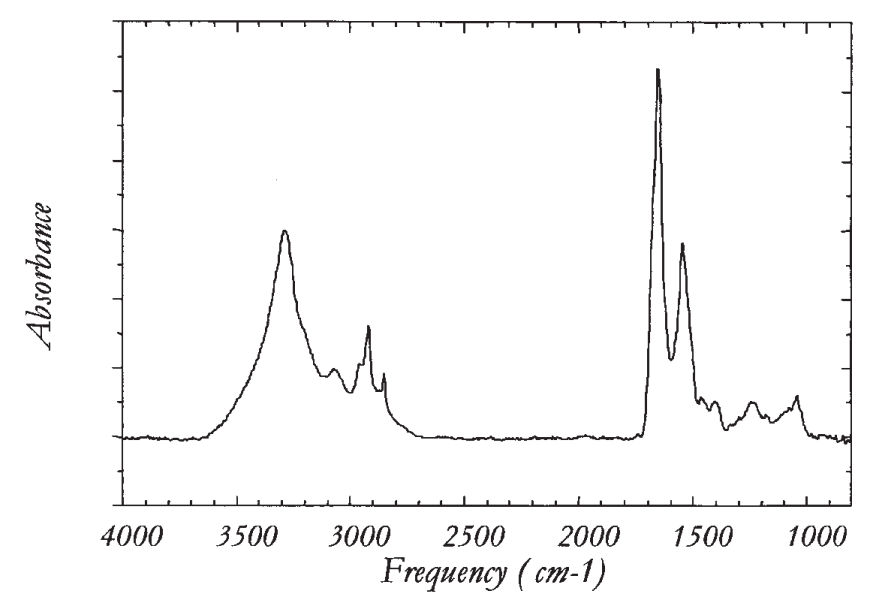

Figure 8. Typical infrared absorbance spectrum of an individual Hybridoma B-cell. (spectrum taken with a $6 \times 6 \mu \mathrm{m}^{2}$ aperture).

use of stains and fixatives. The functional groups could be identified and their concentration profiles mapped with a spatial resolution of a few microns. A sample of a living and dividing cell, whose optical image is shown at the top of figure 9, was mapped and the distributions of protein and lipid are shown at the center and bottom of figure 9. Protein is seen to be concentrated in the centers of each side, and lipids to participate in the build-up of the contractile ring.

It was demonstrated earlier on that necrosis is characterized by the appearance of a $\mathrm{C}=\mathrm{O}$ ester band at $1740 \mathrm{~cm}^{-1}$ [7]. In subsequent experiment, apoptosis (which is the programmed death of the cells) was initiated using an antibody as an inducer. The apoptotic evolution was followed on various individual cells [8]. Figure 10 shows the profile obtained for the $1740 \mathrm{~cm}^{-1}$ and that for the amide II (nucleic acids) band. Clearly, one can notice that the $\mathrm{C}=\mathrm{O}$ ester band develops around the nucleus. This type of experiment gives new insights in the apoptotic process at the molecular level inside an individual cell.

One promising application of synchrotron IR microspectrometry is the study of penetrating agents into the skin. This topic is of high relevant in cosmetics. For such studies, a detailed analysis of the various parts of a skin sample, namely the Stratum Corneum and Stratum Granulosum, has to be made with an improved lateral resolution, since the Stratum Corneum is only a few microns thick. The use of synchrotron radiation for IR microscopy has allowed chemical images of the various functional groups compound of a skin sample to be made, as shown in figure 11 . One can clearly distinguish the two regions making it possible to do depth profiling of any agent penetrating the skin, with high spatial contrast.

\section{Future and perspectives}

Synchrotron infrared microspectrometry is receiving a lot of interest from a wide community of scientists. Its powerful
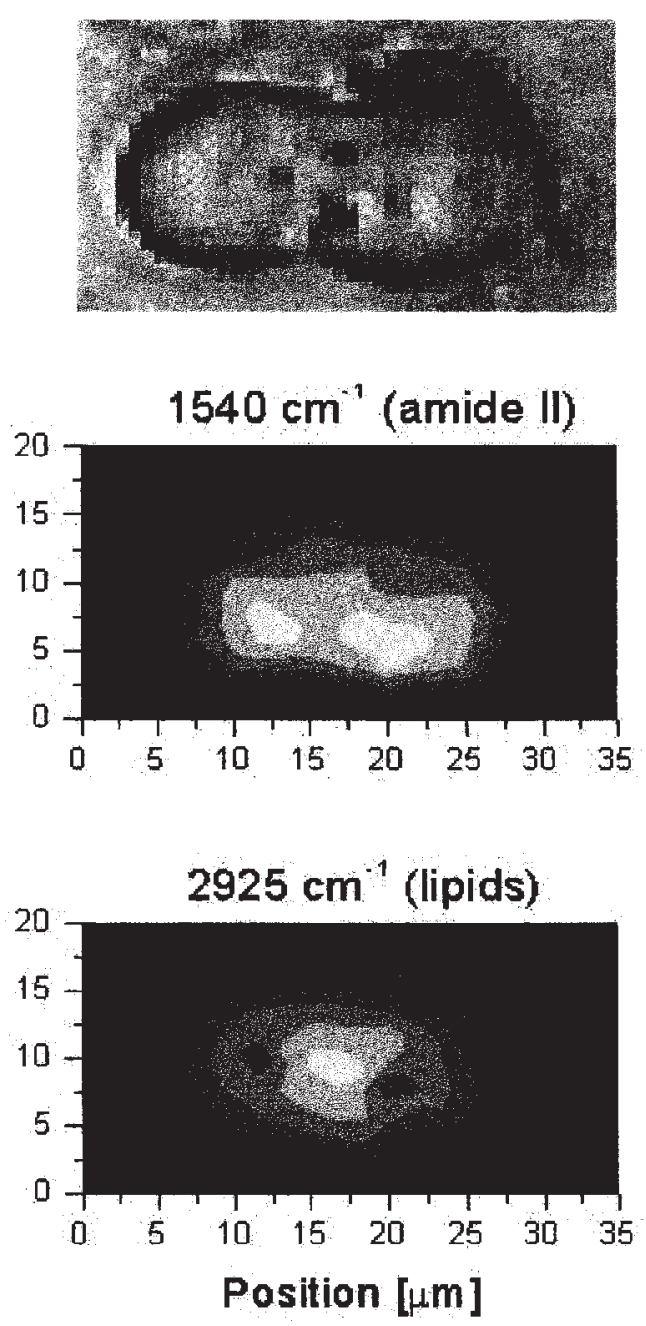

Figure 9. A dividing cell (top) has been mapped. After mapping, the distribution of the amide II band, located at $1540 \mathrm{~cm}^{-1}$, (center) and that of the $\mathrm{CH}_{\mathrm{n}}$ stretch massif (bottom) are not identical. One can notice, on the amide II profiling, the formation of the two nucleus, and on the $\mathrm{CH}_{n}$ massif, the high concentration of lipids in the region where the contractile ring, responsible for the cleavage furrow is located [7].

analytical advantages opens new investigations in new fields. Among them, biologists have found a new tool for investigating the chemical composition and evolution of individual cells. Various synchrotron facilities have included infrared microspectrometry in their development programs. Nowadays, several beamlines are available around the world.

One major step towards a deeper analysis in various research fields, will be to develop in situ investigations. High pressure, high and low temperature cells that can accommodate an automated XY stage are highly desirable. Different areas will benefit from such a development: Geology, to determine the phase diagram of the fluids; Catalysis to follow the reactivity of a single particle; 

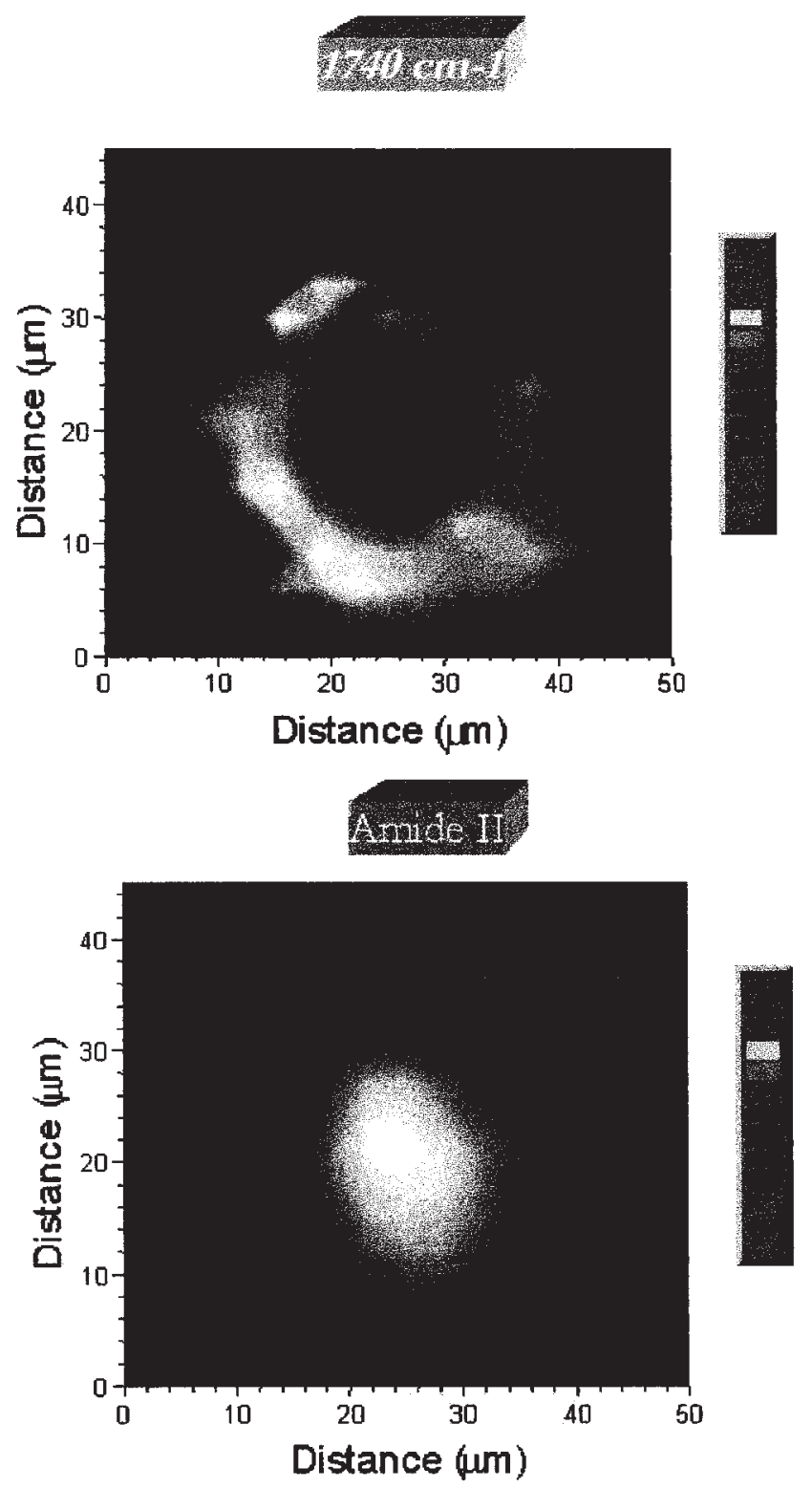

Figure 10. Chemical distribution of the ester band and of the amide II band, on an individual cell during its necrotic stage. The death of this cell has been programmed by adding an anti body. One can clearly note that the $\mathrm{C}=\mathrm{O}$ ester band develops around the nucleus.

Biology to study the time evolution of phenomena induced at a single cell level, or in hair, skin, etc.; Semiconductors, for the study of small areas exhibiting phase transitions.

Besides these applications, new type of experiment has been performed, or is underway, which uses the advantage of the pulse nature of the synchrotron beam [9]. At SUPERACO, there is an UV-storage ring Free Electron Laser (FEL) which is a tunable, coherent and intense (up to $300 \mathrm{~mW}$ ) photon source in the near-UV range $(300-350 \mathrm{~nm})$. Besides,

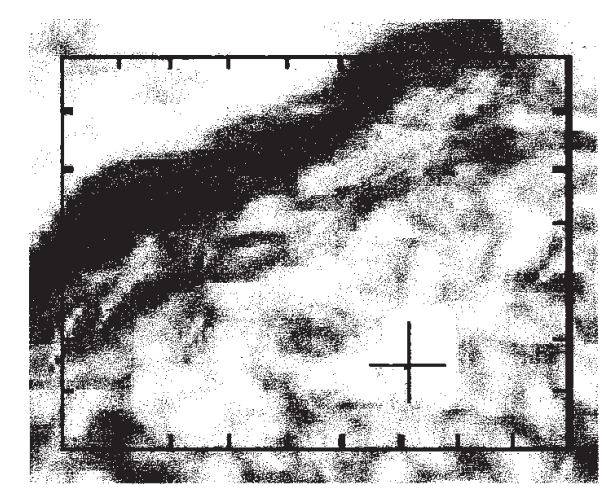

Max.

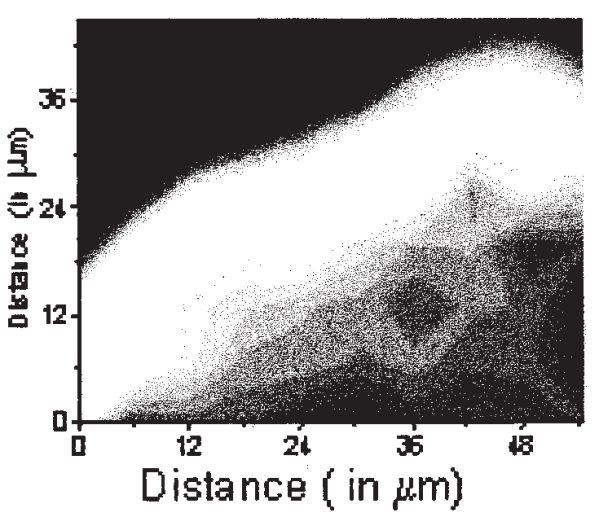

Min.

Figure 11. Top: Optical image of a skin sample. The amide II band has been mapped (bottom), which an aperture of $6 \times 6$ $\mu \mathrm{m}^{2}$, showing an increase concentration of amide functional groups in the Stratum Corneum.

it has the unique feature in being synchronized in a one-toone shot ratio with the Synchrotron Radiation (SR) at the high repetition rate of $8.32 \mathrm{MHz}$. We have recently combined the FEL + SR [10]. This combination appears to be very powerful for the performance of pump-probe timeresolved and/or frequency-resolved experiments on the subns and ns time-scales. In particular, there is a strong scientific case for the combination of the SA5 (MIRAGE) IR Synchrotron Radiation beamline with the UV-FEL, for the performance of transient IR-absorption spectroscopy on FEL-excited samples. This is combined with Fourier transform infrared allowing high lateral resolution. To exemplify the potential of such an arrangement, figure 12 shows the FEL and SR pulses that have been recorded with a pin silicon photodiode for 3 different settings of the optical delay line of +2.4 ns delay (FEL before the SR), of zero delay (perfect temporal overlap) and of -2.5 ns delay (FEL after the SR). The precision in the temporal calibration is of \pm 100 ps. Studies are currently being performed to benefit from this original pump-probe experimental set up, for small domain analysis. 


\section{Summary}

Synchrotron radiation is a bright source of infrared light. Used in conjunction with an IR microscope, enhanced lateral resolution has been achieved, limited only by diffraction. The new analytical possibilities offered by this technique have already been widely exploited in various fields of research. Micron-size particles, of environmental and extraterrestrial relevance, can now be individually analyzed. In geology and biology, high contrast can be achieved, allowing the recording of detailed chemical images. Consequently, fluid inclusions, and individual cells have now been mapped. There are a lot of other potential applications, which will certainly be exploited, in the near future.

Besides these analytical advantages, synchrotron radiation, being a pulsed source, can also offered time resolved (ns to few ps range) investigation of small samples. It is a new analytical tool, easily operated despite the complex environment of a synchrotron facility, which motivates a wide community of new users.

\section{Acknowledgements}

It is a great pleasure to acknowledge the technical and engineering support of the LURE and the NSLS synchrotron facilities, in particular the help of M. Tanguy, J.-P. Marx (LURE) D. Carlson, J. Gallagher, R. Greene, G. Nintzel and D. Lynch (NSLS). Work on the extraterrestrial particles was done in collaboration with L.-H. D'Hendecourt, J.P. Bibring, J. Borg, E. Quirico and P.-I. Raynal (Institut Astrophysique, Orsay, France), while the work on fluid inclusions was done with N. Guihaumou, and that of biology with N. Jamin, J.L. Teillaud, L. Miller and N. Gross. Time resolved experiments are currently being carried out in collaboration with L. Nahon and M.-E. Couprie, from LURE.

The NSLS is supported by the United States Department of Energy under contract DE-AC02-98CH10886.

\section{References}

1. Reffner, J.A.; Martoglio, P.A.; Williams, G.P. Rev. Sci. Instr. 1995, 66, 1298.

2. Carr, G. L.; Reffner, J.; Williams, G. P. Rev. Sci. Instr. 1995, 66,1490

3. Carr, G. L.; Dumas, P.; Hirschmugl, C. J.; Williams, G. P. Il Nuovo Cimento 1998, 20, 375-395.

4. Roy, P.; Mathis, Y. L.; Gerschel, A.; Marx, J. P.; Michaut, J.; Lagarde, B.; Calvani, P. Nucl. Instrum. Methods A 1993, 325, 568.

5. Carr, G. L. et al. to be published, 1999.

6. Raynal, P. I.; Quirico, E.; Borg, J.; Deboffle, D.; Dumas, P.; d'Hendecourt, L.; Bibring, J.P.; Langevin, Y. Mars Special Issue (in press), 1999.
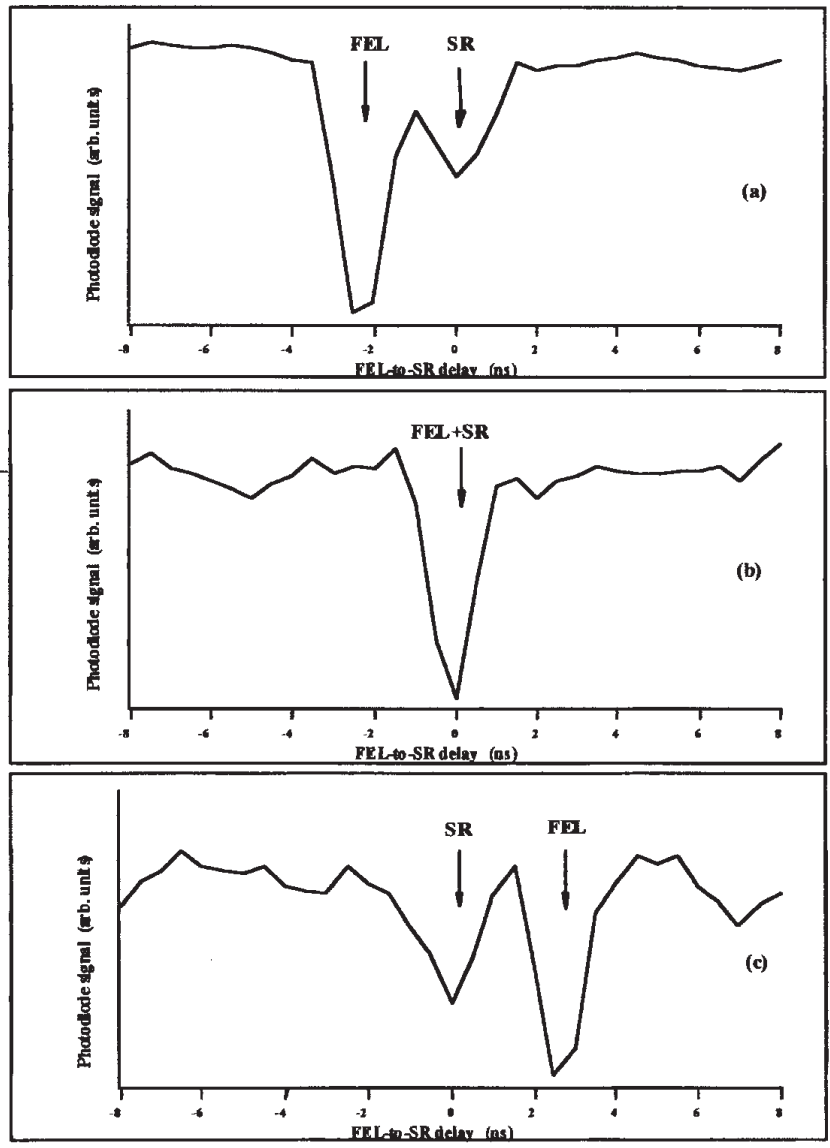

Figure 12. FEL and SR pulses signal as recorded with a pin Silicon photodiode for 3 different settings of the optical delay line: (a) +2.4 ns delay (FEL before the SR); (b) zero delay (perfect temporal overlap); (c) -2.5 ns delay (FEL after the SR). The precision in the temporal calibration is of \pm 100 ps.

7. Jamin, N.; Dumas, P.; Montcuit, J.; Fridman, W.H.; Teillaud, J.L.; Carr, G.L.; Williams, G.P. Proc. of the National Acad. Sci. 1998, 95, 4837 .

8. Teillaud, J.L.; Jamin, N.; Miller, L.; Montcuit, J.; Fridman, W. H.; Carr, G.L.; Williams, G.P.; Dumas, P. to be published.

9. Carr, G.L.; Lobo, R.P.S.M.; Hirschmugl, C.J.; Veigne, J.L.; Reitze, D.H.; Tanner, D.B. "Time resolved spectroscopy using synchrotron infrared pulses," SPIE Conf. Proc. (Ed. P. Dumas and G.P. Williams) 1997, 3153, 127.

10. Nahon, L.; Renault, E.; Couprie, M.E.; Nutarellia, D.; Garzella, D.; Billardon, M.; Carr, G.L.; William, G.P.; Dumas, P. "Two-color experiments combining the UV-Storage Ring Free Electron Laser and the SA5 IR beamline at Super-ACO," SPIE " Accelerator based IR sources and Spectroscopic Applications", Ed. G.L. Carr and P. Dumas, 1999.

11. Guilhaumou, N.; Dumas, P.; Carr, G.L.; Williams, G.P. Applied Spectr. 1998, 52, 1029. 